\section{Serial Competitive RT-PCR Using Multiple Standards}

BioTechniques 33:744-748 (October 2002)

PCR is widely used for the amplification of DNA and reverse-transcribed RNA. In recent years, real-time PCR has been introduced as a new technique for mRNA quantitation. However, because of the high cost of real-time PCR equipment and supplies, especially using custom-made probes, quantitative RT-PCR is a valuable alternative method. Several quantitative PCR protocols describe the use of a single competitive standard $(2,6)$. The aim of the present study was the development of a protocol using several competitive standards of different sizes to quantify gene expression in a one-tube-amplification procedure.

As a model system, endothelin receptor B mRNA was quantified in human endothelial cells. Endothelin-1 is the most potent vasoconstrictor known to date and mediates its effects in cardiovascular physiology and pathophysiology via binding to endothelin receptors type $\mathrm{A}$ or $\mathrm{B}\left(\mathrm{ET}_{\mathrm{A}}\right.$ and $\left.\mathrm{ET}_{\mathrm{B}}\right)$ (4). Endothelial cells express $\mathrm{ET}_{\mathrm{B}}$ receptors only $(3,5)$.

Total RNA from primary cultures of human umbilical vein endothelial cells was isolated as previously described (7). A 702-bp fragment of human $\mathrm{ET}_{\mathrm{B}}$ was cloned from endothelial RNA by RT-PCR (5). Four internally deleted standards $(648,528,438$, and $355 \mathrm{bp})$ were constructed using linker primer PCR (2). Using this approach, target and standards contain the same sense and antisense primer binding sites. We used following primers: $\mathrm{ET}_{\mathrm{B}}$ sense: 5'-CGAGCTGTTGCTTCTTGGAGTAG-3', ET ${ }_{B}$ antisense: 5'-ACGGAAGTTGTCATATCCGTGATC-3', linker 1: 5'-CTTGGAGTAGGGTGGTCTCTGTGGTTCTGG-3', linker 2: 5'-CTTGGAGTAGGCAGTTTTACAAGACAGCAA-3', linker 3: 5'-CTTGGAGTAGGACCTGTGAAATGTTGAGAA-3', and linker 4: 5'-CTTGGAGTAGCCGTCTTTTGCCTGGTCCTT-3'. Standards were cloned into pCR-Script Amp SK ${ }^{+}$Cloning Vector (Stratagene, La Jolla, CA, USA). Standard identity was confirmed by cycle sequencing using ABI PRISM ${ }^{\circledR}$ Dye Terminator Cycle Sequencing Ready Reaction Kit on an automated ABI 373A DNA Sequencer (both from Applied Biosystems, Foster City, CA, USA). Database searches of GenBank ${ }^{\circledR}$ were performed using BLASTN (1). In vitro transcription of cDNA standards was performed using the RNA Transcription Kit (Stratagene), and cRNA was quantified spectrophotometrically at $260 \mathrm{~nm}$. Standard RT after $3 \mathrm{~min}$ at $70^{\circ} \mathrm{C}$ was done for $1 \mathrm{~h}$ at $42^{\circ} \mathrm{C}$ using cRNA random hexamer primers and SuperScript ${ }^{\mathrm{TM}}$ II RNase $\mathrm{H}^{-}$ Reverse Transcriptase (Invitrogen, Paisley, UK). Afterwards, one-fifth of each RT reaction was amplified by PCR with 20 pmol ET $_{\mathrm{B}}$ sense and antisense primers: $1 \mathrm{~min}$ at $95^{\circ} \mathrm{C}, 1 \mathrm{~min}$ at $69^{\circ} \mathrm{C}$, and $1 \mathrm{~min}$ at $72^{\circ} \mathrm{C}$. PCR fragments were separated by agarose gel electrophoresis, stained with ethidium bromide, and documented by photography using Polaroid ${ }^{\circledR}$ film type 665 . The absorbances of sample specific target and standard copies were estimated by a personal densitometer (Molecular Dynamics, Sunnyvale, CA, USA). Densities of PCR fragments were normalized with a correction coefficient and logarithm of quotient of samplespecific and standard PCR fragment density graphically plotted versus the amount of standard RNA.

With our serial RT-PCR using multi- ple standards, a one-tube reaction is sufficient to measure the target copy number (Figure 1). For this purpose, a standard mixture including all four standards was used. In this mixture, the highest copy number was assigned to the smallest standard. Each larger standard was added in a 1:2 dilution to the next smaller standard. This standard mixture was diluted and added to a constant amount of total RNA (250 ng) from human endothelial cells. After RT-PCR and gel electrophoresis, the copy number of the target gene was calculated.

Using this approach, target and standard mixtures of four different internally deleted standards can be transcribed into cDNA and subsequently amplified at the same rate. The $\mathrm{ET}_{\mathrm{B}}$ copy number in human endothelial cells was determined using the serial RT-PCR method using multiple standards ( $\overline{\mathrm{x}} \pm \mathrm{SEM} / \mu \mathrm{g}$ RNA: $1917.8 \pm 36.5 ; n=4)$. The method is highly reproducible.

To test the validity of the approach, we compared the well-established competitive approach and our new serial RT-PCR using multiple standards (Figure 2A). An identical copy number of target and standard cDNA was used in each approach. Both competitive and serial RT-PCR using multiple standards were carried out in a parallel manner. We found no significant difference in deviation between the two approaches. In both cases, a difference of approxi-

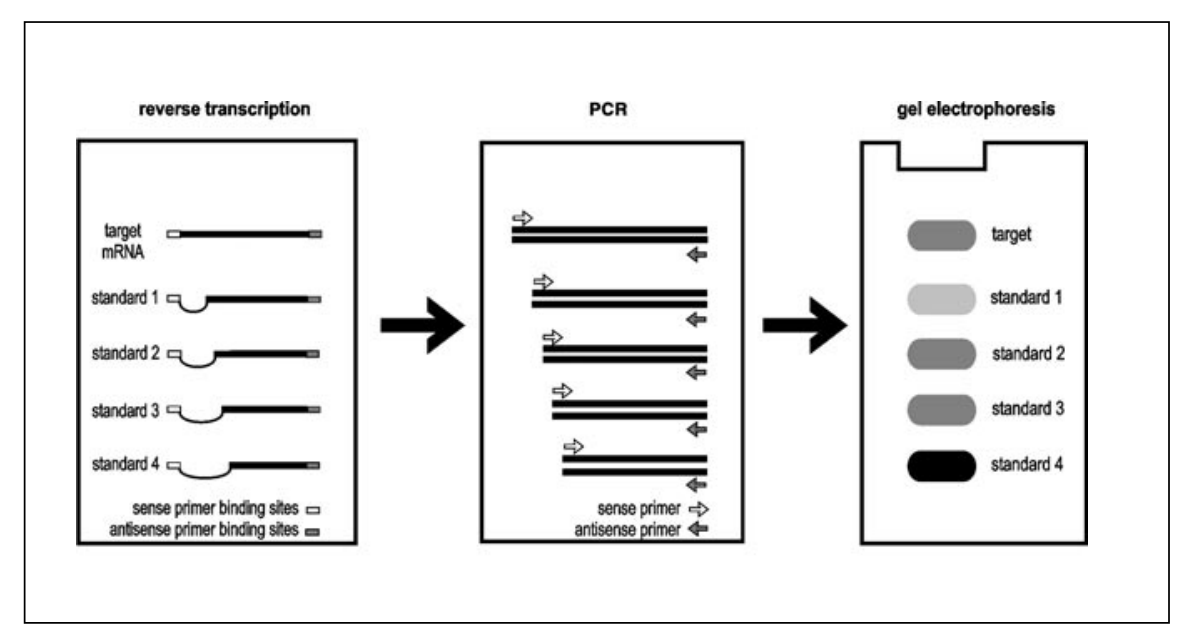

Figure 1. Serial RT-PCR strategy using multiple standards. The methods used are in contrast to wellestablished competitive RT-PCR: not serial dilutions of one standard in several tubes, but dilutions of serially deleted standards carrying identical primer binding sites in a single tube. Four standards, each different in size and concentration, are added to a known amount of total RNA from a cell preparation or biopsy. PCR products are separated using standard agarose gel electrophoresis and stained with ethidium bromide. Afterwards, mRNA expression of target gene can be quantified densitometrically. 


\section{A

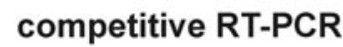

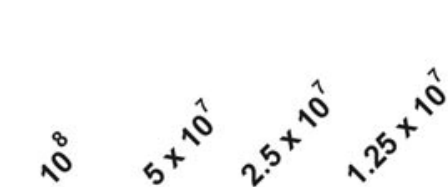

serial multistandardassisted RT-PCR
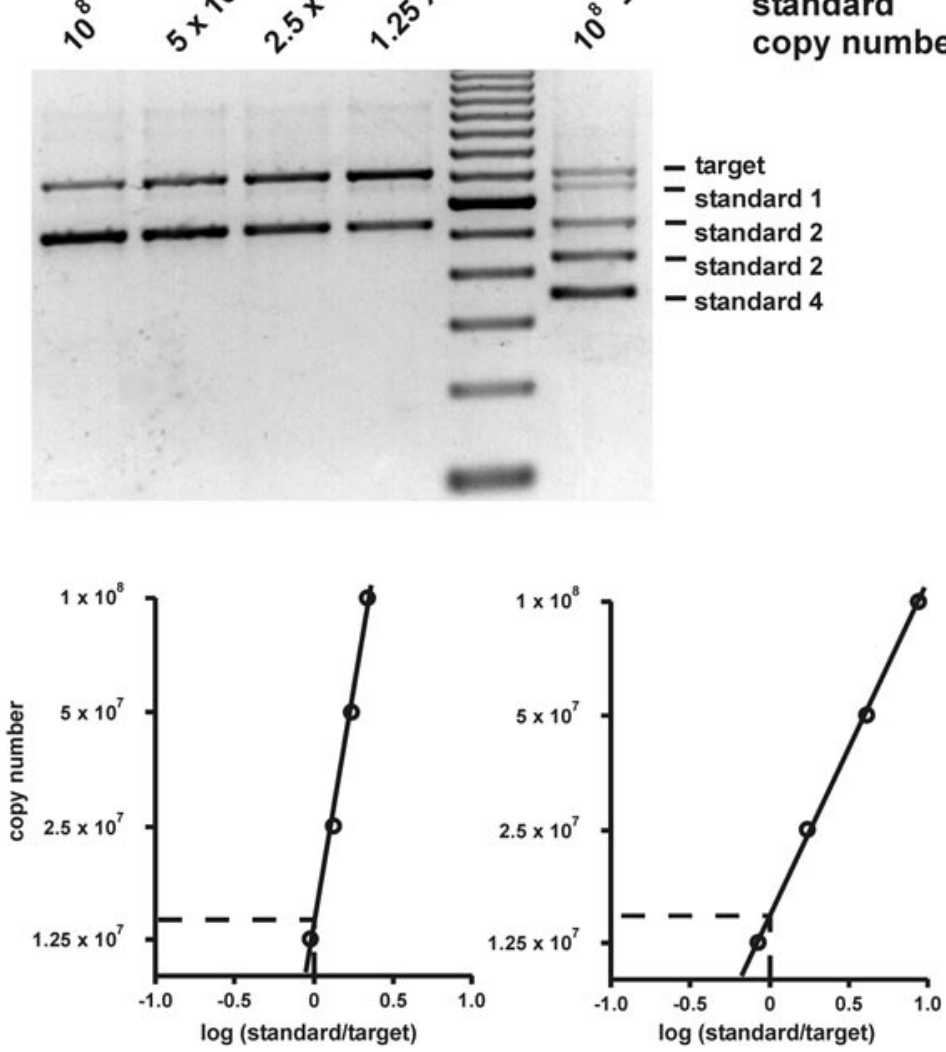

B

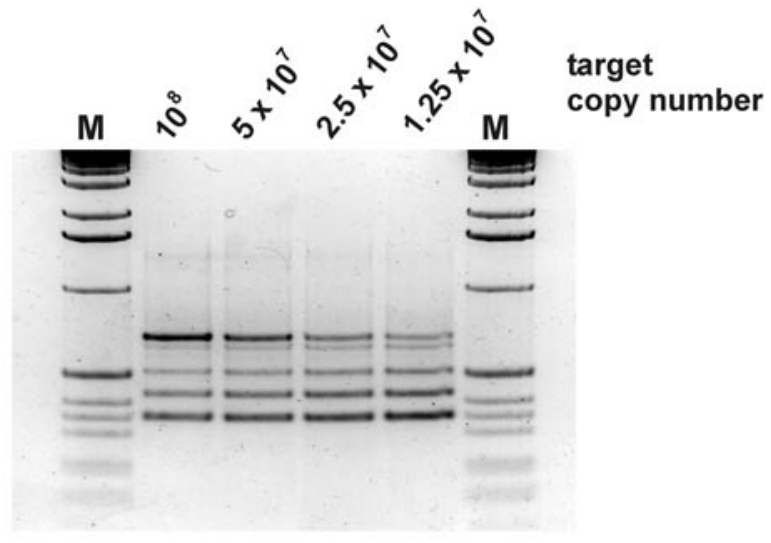

Figure 2. Comparison of competitive and serial RT-PCR using multiple standards. (A) Electrophoretic separation (upper panel) and determination of copy number (lower panel) are demonstrated, comparing competitive and serial RT-PCR using multiple standards. A known copy number of sample-specific target cDNA and standard (1:2 mixture) was added to each reaction. Afterwards, both quantitative RT-PCR approaches were carried out in a parallel manner $(n=13)$. Neither the PCR protocols nor the copy number calculations differed. Both applications gave equal results. (B) Different amounts of target copy numbers were amplified by serial RT-PCR using multiple standards and separated by gel electrophoresis. Copy number did not interfere with the results of quantitation by serial RT-PCR using multiple standards. mately $10 \%$ from the predicted target fragment was calculated. The difference between the empirically, PCRbased, calculated target concentration and the theoretically, densitometerbased measured concentration was $12.4 \% \pm 1.8(\overline{\mathrm{x}} \pm \mathrm{SEM} ; n=16)$ for competitive PCR, $8.9 \% \pm 2.0(\overline{\mathrm{x}} \pm \mathrm{SEM} ; n=$ 13) for multistandard-assisted RT-PCR. Different amounts of target copy numbers did not interfere with the results of quantitation by serial RT-PCR using multiple standards (Figure 2B).

With three dilutions of 1:2, the concentration of the highest and lowest standards differed by a factor of 8 . The working range may be even bigger. If the target gene expression falls outside the range of the original dilution, then an additional dilution might be needed. The sensitivity of the competitive and the serial competitive approaches was not different. Furthermore, different numbers of cycles did not interfere with the results. However, the reliability of this approach has to be tested very carefully for every specific application.

In summary, the competitive and the serial, multiple-standard approaches of quantitative RT-PCR were shown to be highly sensitive and specific. We have generated a novel application of the competitive RT-PCR technique, the serial RT-PCR using multiple standards. This new approach of quantitative RTPCR could be used in a variety of experimental and clinical applications. Even while establishing that the technique could initially take longer to generate additional standards and adapt them to the protocol, this method has several advantages. First, it can be performed in every laboratory with no need for any additional equipment. Second, all components are identical with chemicals that are already in use for standard PCR. Third, procedures can be more easily automated during PCR and the calculation of copy number. Furthermore, this one-tube technique enables the laboratory staff to quantify gene expression on a lower-cost level and in shorter time. In addition, the method allows the efficient quantitation of mRNA expression even in microbiopsies (from 5 to $2 \mathrm{mg}$ tissue). Finally, these advantages do not interfere with the typical high specificity and sensitivity of quantitative RT-PCR. 


\section{REFERENCES}

1.Altschul, S.F., W. Gish, W. Miller, E.W. Myers, and D.J. Lipman. 1990. Basic local alignment search tool. J. Mol. Biol. 215:403410.

2.Forster, E. 1994. An improved general method to generate internal standards for competitive PCR. BioTechniques 16:18-20.

3.Heinroth-Hoffmann, I., M. Vogelsang, P. Schiewe, H. Morawietz, J. Holtz, K. Ponicke, and O.E. Brodde. 1998. Mechanism of $\mathrm{ET}_{\mathrm{A}}$-receptor stimulation-induced increases in intracellular $\mathrm{Ca}^{2+}$ in SK-N-MC cells. Br. J. Pharmacol. 125:1202-1211.

4.Miyauchi, T. and T. Masaki. 1999. Pathophysiology of endothelin in the cardiovascular system. Annu. Rev. Physiol. 61:391-415.

5.Ogawa, Y., K. Nakao, H. Arai, O. Nakagawa, K. Hosoda, S. Suga, S. Nakanishi, and H. Imura. 1991. Molecular cloning of a non-isopeptide-selective human endothelin receptor. Biochem. Biophys. Res. Commun. 178:248-255

6.Orlando, C., P. Pinzani, and M. Pazzagli. 1998. Developments in quantitative PCR. Clin. Chem. Lab. Med. 36:255-269.

7.Schubert, A., M. Cattaruzza, M. Hecker, D. Darmer, J. Holtz, and H. Morawietz. 2000. Shear stress-dependent regulation of the human $\beta$-tubulin folding cofactor $D$ gene. Circ. Res. 87:1188-1194.

We are grateful to J. Holtz for critically reading the manuscript and $R$. Gall and $R$. Busath for excellent technical assistance. Address correspondence to Dr. Henning Morawietz, Institute of Pathophysiology, Martin Luther University Halle-Wittenberg, Magdeburger Str. 18, D-06097 Halle, Germany.e-mail: henning.morawietz@medizin.uni-halle.de

Received 6 March 2002; accepted 3 July 2002.

\section{Marten Szibor and Henning Morawietz \\ Martin Luther University Halle-Wittenberg \\ Halle, Germany}

For reprints of this or any other article, contact Reprints@BioTechniques.com

\section{Simultaneous Detection of Co-Segregating Missense Mutations of the Toll-Like Receptor 4}

BioTechniques 33:748-754 (October 2002)

Cells of the innate immune system sense host invasion by detecting structural determinants that are broadly conserved among pathogens of a given phylogenetic group (4). The lipopolysaccharides (LPSs or endotoxins) that decorate the outer membrane of Gramnegative bacteria are excellent examples of such determinants. In response to minute concentrations of LPS derived from certain Gram-negative bacteria, macrophages secrete cytokines such as tumor necrosis factor (TNF), interleukin-1 (IL-1), and IL-6, which contribute to the containment of the infection and help to initiate a specific immune response. Overstimulation of the innate immune system through this channel can lead to acute systemic inflammation and shock $(2,11)$.

Evidence for genetic control of response to LPS was provided in studies of $\mathrm{C} 3 \mathrm{H} / \mathrm{HeJ}(9)$ and $\mathrm{C} 57 \mathrm{BL} / 10 \mathrm{ScCr}(3)$ mouse strains, which are highly resistant to the deleterious biologic effects of LPS. This resistance is due to defects in the gene encoding the transmembrane Toll-like receptor 4 (TLR4) (7).

Recently, two co-segregating missense mutations, Asp299Gly and Thr399Ile, which are caused by an Ato-G transition at nucleotide 2326 and a C-to-T transition at nucleotide 2626, were identified in a group of individuals with LPS-hyporesponsiveness to inhaled LPS. Transfection experiments demonstrated that the conversion of Asp299 to Gly interrupts TLR4-mediated LPS signaling, leading to a decreased response to incorporated LPS associated with increased susceptibility to Gram-negative bacteremia. For Thr399Ile the data are not yet clear and still await confirmation (1). Both polymorphisms in the TLR4 gene could constitute an important prognostic factor in patients with generalized bacteremia or septic shock.

Lorenz et al. (6) found that the TLR4 mutations mentioned above in either transplant recipients or donors or both are associated with a reduced risk of acute graft versus host disease (GVHD) but may increase the risk for infection with Gram-negative bacteremia. However, the associations were not statistically significant, probably due to small sample size (6).

It has been reported that TLR4 is expressed by macrophages in murine and human lipid atherosclerotic lesions, suggesting that TLR4 may provide the pathophysical link between lipids, infection/inflammation, and atherosclerosis. Thus, it is tempting to speculate that the co-segregating missense mutations Asp299Gly and Thr399Ile may be involved in the pathogenesis of atherosclerosis (12).

To address this question, studies with large numbers of patients will be required, and fast and economic assays that can be performed with standard PCR equipment are highly desirable.

Therefore, we developed a mutagenically separated multiplex PCR for the simultaneous detection of both known mutations in the TLR4 gene. The mutagenically separated PCR we developed is a single-tube, PCR-based technique that relies on allele-specific primers that differ in length by $8 \mathrm{bp}$. Base mismatches in the allele-specific primers introduce deliberate differences into the allelic PCR products that minimize cross-reactions of the PCR products in subsequent cycles. Because of the 8-bp length difference, the alleles are easily discernible by electrophoresis on high-resolution gels.

We first determined the genotypes in the TLR4 gene in 52 individuals using a published protocol (6) in which TLR4 sequences are amplified by PCR and the mutations are detected by restriction enzyme digestion. Aliquots of 5 $\mu \mathrm{L}$ PCR product were digested with NcoI (TLR4 Asp299Gly) and HinfI (TLR4 thr399Ile) at $37^{\circ} \mathrm{C}$ overnight and electrophoresed on Novex 6\% polyacrylamide gels (Invitrogen, Carlsbad, CA, USA). DNA was extracted from citrated venous blood using the Purgene ${ }^{\circledR}$ DNA Isolation Kit (Gentra System, Minneapolis, MN, USA). Generally, the blood was frozen at $-20^{\circ} \mathrm{C}$ within 3-5 h after sampling. For each DNA sample, the quality was controlled by agarose gel electrophoresis. 